\title{
A case of isolated atrioventricular discordance
}

\author{
R CALABRO, B MARINO, F MARSICO
}

From the Division of Cardiology, Ospedale Pausilipon, Naples; and Institute of Cardiology, Catholic University, Rome, Italy

SUMMARY A case of isolated atrioventricular discordance in situs solitus is described.

The importance of a well defined situs and of the presence of both ventricles in making this diagnosis is underlined and previous published reports reviewed.

It is suggested that the haemodynamic concept of isolated atrioventricular discordance cannot be described only by the spatial relation between the ventricles (isolated ventricular inversion), but it should be indicted by the pattern of the connection between various flows, as shown by hearts with a normal relation between the ventricles and crossed atrioventricular connection.

We consider that " $\mathrm{A}-\mathrm{V}$ discordance with ventriculoarterial concordance and normally related great arteries"' is the best way to describe the anatomical and the haemodynamic pattern in so called isolated ventricular inversion.

In this paper we report a new example of this rare anomaly which we call "isolated atrioventricular discordance". To our knowledge, this is the first reported case in which a straddling left atrioventricular valve was present.

\section{Case report}

A 2-month-old female infant (birthweight $2.5 \mathrm{~kg}$ ) was admitted with dyspnoea and cyanosis which had been present from birth.

A xiphoid impulse was present and a grade $3 / 6$ harsh early and mid-systolic murmur was heard at the left lower sternum; the second heart sound was normal. The peripheral pulses were diminished and the liver was much enlarged. Chest $x$-ray film showed a left cardiac apex, cardiomegaly, and increased pulmonary flow. The electrocardiogram showed normal sinus rhythm, left atrial enlargement, and a QRS axis at $+140^{\circ}$ in the frontal plane. An RS pattern in all the praecordial leads was present. The vectorcardiogram showed a figure-of-eight inscription in the frontal plane with clockwise rotation and inferior and leftward directed initial forces; in the horizontal plane, counterclockwise rotation was present, with most of the loop in the posterior and right quadrant. The $0.01^{\mathrm{s}}$ vector was oriented anteriorly and to the left.

ANATOMICAL DESCRIPTION

Laevocardia and solitus visceroatrial situs were pres- ent, with normal atrial morphology and the normal relation between the atrial chambers. Systemic and pulmonary connections were normal. The atrioventricular connection was discordant with two separated atrioventricular valves, the left of which was straddling. The right atrium was connected to a right sided and morphologically left ventricle through a bicuspid valve (Fig. 1A). The subvalvular apparatus was normal. A normal-sized ventricle was connected to a right sided and posterior aorta (Fig. 1B). Fibrous continuity between the anteromedial leaflet of the right atrioventricular valve and the aortic cusps was documented. A fibromuscular membrane obstructing the aortic outflow tract was present (Fig. 1B). The ventricular septum was abnormally orientated, so that its posterior aspect was to the left of its anterior aspect; a perimembranous ventricular septal defect was present (Fig. 1B). Some chordae from the left atrioventricular valve had their insertion on the base of the septal defect on its right side. The left atrium was connected to a left sided, morphologically right ventricle through a tricuspid valve (Fig. 2A). The normal sized atrioventricular valve annulus was shifted medially and to the right but did not override the ventricular septum. The anterior leaflet of the left tricuspid atrioventricular valve had its insertion on the anterior papillary muscle and on the left side of the septum by some chordae tendineae. The posterior leaflet had its insertion on two posterior papillary muscles. The septal leaflet inserted directly on the septum with some chordae tendineae on the left side, and some, through the ventricular septal defect, on the right side of the ventricular septum (Fig. 2A). The morphologically right ventricle was smaller than the left, had a well-developed "crista supraventricularis", 


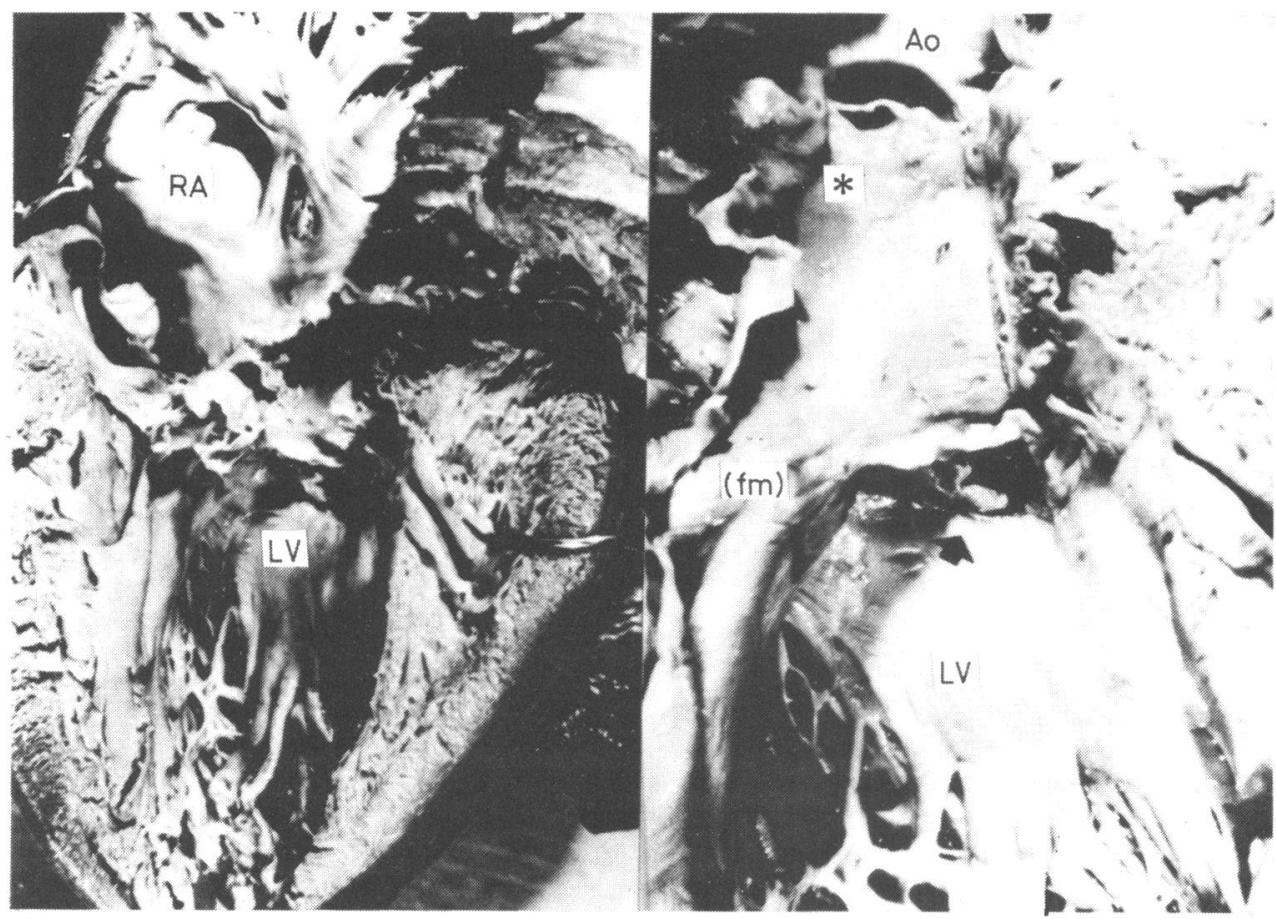

(A)

(B)

Fig. 1 (A) Right atrioventricular connection: the right atrium $(R A)$ is connected to a morphologically left ventricle $(L V)$ through a bicuspid valve. $(B)$ This ventricle is normally connected to a right sided and posterior aorta (Ao). Fibrous continuity between the atrioventricular valve and the aortic cusps ( $\left.{ }^{\star}\right)$. A fibromuscular membrane $(\mathrm{fm})$ is present in the subaortic outflow tract. Through a perimembranous ventricular septal defect there are some chordae of the left sided atrioventricular valve (arrow).

and was connected to the pulmonary artery with an infundibulum (Fig. 2B). Coarctation of the aorta and persistent ductus arteriosus were present.

\section{Discussion}

The final diagnosis in our case is: situs solitus, atrioventricular discordance, with straddling but not overriding left atrioventricular valve, left sided and anterior morphologically right ventricle, right sided and posterior morphologically left ventricle, ventriculoarterial concordance and normally related great arteries, fibromuscular subaortic stenosis, coartation of the aorta, and persistent ductus arteriosus.

Isolated atrioventricular discordance requires the presence of a well-defined situs (solitus or inversus) and of both ventricles, regardless of their spatial relation.

In our view, some published cases of isolated ventricular inversion do not fit this description, because they are univentricular hearts, ${ }^{2-4}$ but an isolated atrioventricular discordance is present in the other published cases described as isolated ventricular inversion,,$^{5-8}$ and in Attie et al.'s second case reported as crossed atrioventricular connection with atrioventricular discordance and normal connection and relation between the great vessels. ${ }^{9}$

In fact, in isolated atrioventricular discordance, the mode of atrioventricular connection may be through two separate atrioventricular valves, one common atrioventricular valve,${ }^{8}$ or a criss-cross situation. ${ }^{9}$ The relation between the two ventricles may vary. Usually the position of the ventricular septum and the relation between the two ventricles are inverted compared with the atrial situs. This is not true when a criss-cross situation is present or when the ventricular septum is horizontal, with a superoinferior relation between the ventricles. In this anomaly the ventriculoarterial connection must be concordant, with normally related great arteries.

The most frequently occurring infundibular morphology is represented by mitral aortic continuity and muscular discontinuity between the tricuspid valve and the pulmonary artery. 


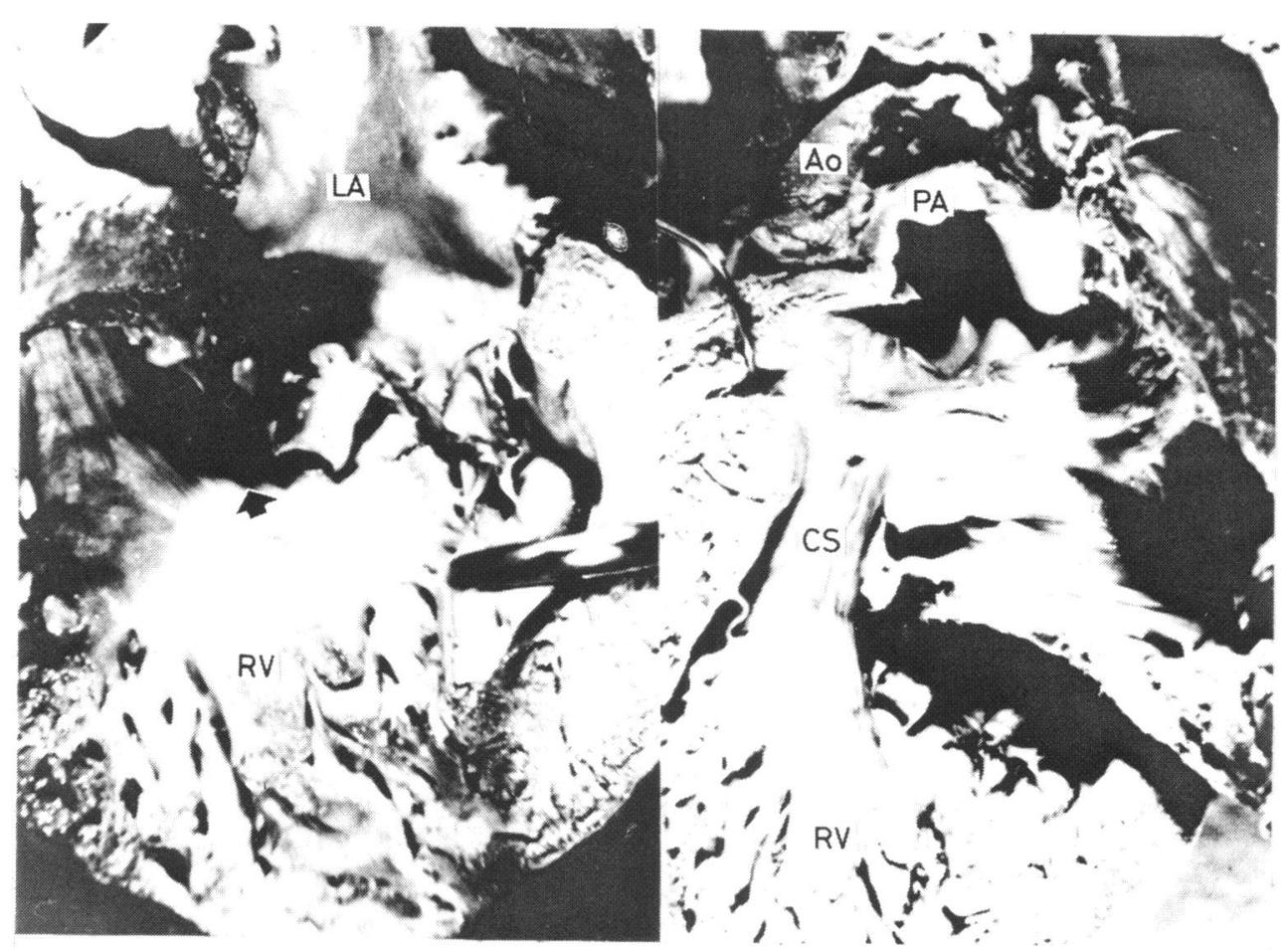

(A)

(B)

Fig. 2 (A) Left atrioventricular connection: the left atrium $(L A)$ is connected to a morphologically right ventricle $(R V)$ through a tricuspid valve. Through the perimembranous ventricular septal defect the septal leaflet is inserted directly on the septum. with chordae on the left and on the right (arrow). (B) The "crista supraventricularis" (CS) is well developed and a muscular discontinuity is present between right ventricle $(R V)$ and pulmonary artery $(P A)$.

The most frequently associated anomalies are left atrioventricular valve (tricuspid) stenosis, with consequent right ventricular hypoplasia when situs solitus is present and right ventricular outflow tract obstruction when situs inversus is present. Other reported anomalies are ventricular septal defects of various types, ${ }^{7}$ aortic stenosis, ${ }^{5}$ coarctation of the aorta, ${ }^{8}$ and persistent ductus arteriosus. ${ }^{7}$ From a clinical standpoint, the vectocardiographic finding of anterior and leftward early vectorial forces (when situs solitus with laevocardia is present), or their anteriorly and rightward orientation (when situs inversus with dextrocardia is present), may be significant. This abnormal orientation is the result of the anomalous position of the ventricular septum.

The final diagnosis of this anomaly may be made only by cardiac catheterisation and angiocardiographic data.

We think that, in order to describe and define cardiac anomalies correctly, the intracardiac circulation should be stressed first and, then the relation between the chambers. Therefore, we suggest that the haemodynamic concept of isolated atrioventricular discordance cannot be described only by the spatial relation between the ventricles, but it should be indicated by the pattern of the connection between the various flows. This is supported by the reported cases of hearts with inverted spatial relation between the ventricles, but with crossed atrioventricular connection and, consequently, with normal circulation. ${ }^{9}$

\section{References}

1 Shinebourne EA, Macartney FJ, Anderson RH. Sequential chamber localization. Logical approach to diagnosis in congenital heart disease. $\mathrm{Br} \mathrm{Heart} \mathcal{F}$ 1976; 38: 327-40.

2 Tandon R, Moller JH, Edwards JE. Ventricular inversion associated with normally related great vessels. Chest 1975; 67: 98-100.

3 Freedom RM, Nanton M, Dische MR. Isolated ventricular inversion with double inlet left ventricle. Eur $\mathcal{F}$ Cardiol 1977; 5: 63-86.

4 Dunkman WB, Perloff JK, Roberts WC. Ventricular inversion without transposition of great arteries: a rarity found in association with atresia of the left-sided (tricus- 
pid) atrioventricular valve. Am $\mathcal{F}$ Cardiol 1977; 39: 22631.

5 Van Praagh R, Van Praagh S. Isolated ventricular inversion: a consideration of morphogenesis, definition and diagnosis of non transposed and transposed great arteries. Am $\mathcal{F}$ Cardiol 1966; 17: 395-406.

6 de la Cruz MV, Amoedo M, Rivera E, Attie F. Arterioventricular relations and their classification. Two specimens of arterioventricular discordance and review of published reports. Br Heart $\mathcal{F}$ 1974; 36: 539-53.

7 Quero Jiménez M, Raposo-Sonnenfeld I. Isolated ventricular inversion with situs solitus. Br Heart $\mathcal{F}$ 1975; 37: 293-304.
8 Hazan E, Baillot F, Rey C, Dupuis C. Isolated ventricular discordance and complete atrioventricular canal in situs inversus: report of successful surgical repair. Am $\mathcal{F}$ Cardiol 1977; 40: 463-6.

9 Attie F, Munoz-Castellanos L, Ovseyevitz J, et al. Crossed atrioventricular connections. Am Heart f 1980, 99: 16372.

Requests for reprints to Dr Raffaele Calabrò, Via R Bracco 71, Naples, Italy. 